\title{
Knowledge and Application of Cardiovascular Disease Prevention Guidelines among Family Physicians: A Cross-sectional Study
}

\author{
Oleksii Korzh $^{1 * \mathbb{D}}$, Yevgenii Nikolenko ${ }^{2}$, Anna Titkova ${ }^{1} \mathbb{D}$, Yelizaveta Lavrova ${ }^{1}$, Kira Vovk $\mathbb{D}^{2}$ \\ ${ }^{1}$ Department of General Practice-Family Medicine, Kharkiv Medical Academy of Postgraduate Education, Kharkiv, Ukraine; \\ ${ }^{2}$ Department of General Practice-Family Medicine, V. N. Karazin Kharkiv National University, Kharkiv, Ukraine
}

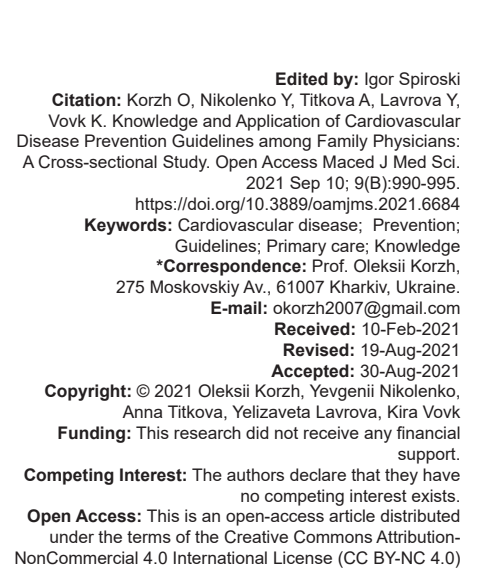

\section{Introduction}

Organizing broad cardiovascular disease (CVD) prevention is still a huge challenge in which general practice plays an important role in promoting a healthy lifestyle across the population [1], [2]. Family physicians (FPs) are at the frontline of providing preventive care, and not surprisingly, studies have identified numerous challenges. It is important to realize that the responsibility of the FP extends beyond the clinical practice [3].

Primary care can only fulfill this expectation, if evidence-based prevention and treatment are provided for all eligible patients at reasonable costs. In 2016, a new version of the European Guidelines on Cardiovascular Prevention was released [4]. The new guideline provides practical tools that can help in the communication about CV risk. They can be used in all populations, irrespective of baseline risk.

One issue that stands out as particularly complex is decision-making about preventive medications. This requires weighing up the potential of future benefits, which is difficult to quantify for an individual, against short-term risk of harm due to adverse effects of medications in the context of decreasing life expectancy and overall health [5]. In a qualitative study, FPs expressed feeling "under pressure" from clinical guidelines to prescribe preventive medicines despite acknowledging that potential harms of side effects of preventive medication and polypharmacy may outweigh future risk reduction [6].

Cardiovascular preventive care in Ukraine is mainly delivered by both family doctors and cardiologists who are employed at the state medical services (hospitals and outpatient clinics) and in private medical facilities. Guidance in prevention is obtained through the guidelines of the European Society of Cardiology. These guidelines should be implemented in the medical services after adaptation to the national resources in Ukraine [7].

This study assesses the determinants of FPs' knowledge and application of cardiovascular preventive management guidelines at primary health-care settings in Ukraine. 


\section{Methods}

This is a cross-sectional study conducted among the 226 FP in Kharkiv Region. The questionnaire was distributed through e-mail over three waves between April 2019 and November 2019 until 226 completed questionnaires were collected.

The target population of the study was all physicians working at primary health-care centers. Hence, no sample size calculation was required as all the physicians were surveyed without random sampling taking place.

The study was conducted according international standards of bioethics and the recommendations of the Committee on Bioethics of the Ministry of Health of Ukraine. This study was approved by the Ethics Commission of the Kharkiv Medical Academy of Postgraduate Education of the Ministry of Health of Ukraine (Kharkiv, UA).

The participants were informed that their participation is voluntary and they are free to refuse the participation in the study or withdraw at any stage without being asked about the reasons or being persuaded. The participants were informed that their refusal or withdrawal from the study participation has no consequences and their information will be confidential. The participants gave oral consent to participate in this study.

The research tool was a self-administrated questionnaire designed by theauthors. Thequestionnaire was designed through an extensive literature search, expert opinions, and use of different manuals on the practice of CVD prevention management. The research tool went through phases of validation until we arrived at the final version that was distributed to the physicians. The first phase of validation was face validation by three experts (consultant physicians) in the field. The questionnaire was adjusted according to the experts' feedback. The process was repeated until the final form was accepted and approved by the three experts.

The second phase consisted of a test-retest process where the questionnaire was distributed to a small sample of 26 physicians from the outpatient clinics that were not included in the final questionnaire distribution. The questionnaire was redistributed again after 2 weeks. Measures of agreements between the answers in the first and second distribution were conducted using intraclass correlation. The items that showed a low agreement were excluded from the questionnaire. The minimum acceptable level for agreement is $75 \%$ [8]. No items were found to have $<75 \%$ agreement. Therefore, no items were removed from the final version.

The scoring method included two types of questions. The first type consists of questions that can only have one correct answer. These questions have a binary scoring method (1 mark for the correct answers and 0 mark for incorrect answers). The second type of questions consisted of answers that can be scaled in terms of correctness. These questions do not have incorrect answers. The best answer among the correct answers scored the highest mark, and the least correct answer scored the lowest mark. The scale used 1 mark step between the answers for this type. For example, if a question had five answers, then the best answer would have 5 marks, the second-best answer would have 4 , and so on, with the least correct answer having a single mark.

The maximum score for the questions that measured the physicians' knowledge about CVD prevention equaled 13 marks. The maximum score for the physicians' application of CVD prevention equaled 21 marks. The cutoff point that was considered as the minimum acceptable score was $70 \%$.

The sociodemographic characteristics of the participants are described in the results of the scores for the items that measured the physicians' knowledge and application of CVD prevention. One-sample t-test is used to test the differences between the scores and the proposed minimum acceptable score. Two independent samples t-test was carried out to test any differences between two groups within the same variable, for example, males' score versus females' score. Oneway analysis of variance is used for the variables that contained three or more levels such as job title. The determinants for knowledge among physicians were assessed using multiple linear regression. The final results are presented in the next section.

There was no involvement of patients or the public in establishing the research questions of this study or defining the outcome measures. Likewise, patients/ the public were not involved in the design, recruitment, or conduct of this study. Patients or the public were not consulted regarding the interpretation or writing of the results. We do not have plans to disseminate the results of this study directly to participants. However, the data will be included in presentations given by the authors to a wide range of audiences.

\section{Results}

Table 1 shows the sociodemographic characteristics of the participants. The mean age was $45.36 \pm 5.82$ years. Females represented $73 \%$ of the sample. Most respondents $(87.1 \%)$ practiced for more than 5 years.

The guidelines for CVD preventive management used by the participants are presented in Table 2 . The majority of the participants $(67.3 \%)$ use the European guidelines. The Ukrainian guidelines 
Table 1: Sociodemographic and job aspect characteristics of the participating physicians $(n=226)$

\begin{tabular}{lll}
\hline Variable & Category & Frequency $(\%)$ \\
\hline Age group & Young $(<30)$ & $87(38.5)$ \\
& Middle aged $(30-60)$ & $116(51.3)$ \\
Gender & Senior $(>60)$ & $23(10.2)$ \\
& Male & $61(27)$ \\
Length of practice & Female & $165(73)$ \\
& $<5$ years & $29(12.9)$ \\
& $5-10$ years & $85(37.6)$ \\
Professional status & $11-15$ years & $74(32.7)$ \\
& $\geq 16$ years & $38(16.8)$ \\
& Family physicians & $165(73)$ \\
& Resident & $47(20.8)$ \\
\hline
\end{tabular}

came in the second place $(25.7 \%)$, other guidelines received $7 \%$ of the answers.

Table 2: Guidelines for CVD prevention management used by the participating physicians $(n=226)$

\begin{tabular}{ll}
\hline Guideline & Frequency (\%) \\
\hline European CVD prevention guideline & $152(67.3)$ \\
Ukrainian CVD prevention guideline & $58(25.7)$ \\
Other guidelines & $16(7)$ \\
\hline CVD: Cardiovasculardisease. &
\end{tabular}

The participants were asked about the barriers to using CVD prevention. The participants were allowed to choose more than one barrier. Lack of knowledge of the CVD preventive care was considered the biggest barrier $(62.8 \%)$. Lack of counseling skills was the second major barrier (37.9\%). Subjectivity of the questions was considered to be the third barrier (32.6\%).

In the results for three questions of binary output (correct vs. incorrect) that measured knowledge of guidelines, two questions show less than $70 \%$ correct answers. First question: "How many steps do you know in terms of preventing cardiovascular disease?" received only $53.8 \%$ of correct answers. The second question "which of the following is not an item of CVD prevention?" received $67.1 \%$ correct answers. However, this percentage is still below the minimum acceptable level. The only question that received $70 \%$ or more correct answers was the question about the criteria used to classify patients with CVD (76.5\%).

The next question in the knowledge of guidelines was about the percentage of physicians prescribing statins for their CVD prevention. This question was scaled. As described in the methodology section, the least correct answer scored 1 mark and the best correct answer scored 5 marks. For this question, $<20 \%$ was the least correct answer, and as the percentage categories increased the mark increased. Hence, the best correct answer was $81 \%-100 \%$. Most of the participants preferred the middle ground where they use statins between $41 \%$ and $60 \% ; 42.3 \%$ of the asked physicians preferred this amount of usage. The best usage per guideline was $81 \%-100 \%$; however, this category received the lowest percentage of selections with only $4.6 \%$.

The last question that measured knowledge of guidelines asked the physicians about the percentage of their use of antihypertensive medication. The least percentage corresponded with the highest mark (5 marks) and the highest percentage corresponded with the lowest mark (1 mark). The least two correct answers had the highest percentages of answers (31.4\% and $29.8 \%$, respectively). The best correct answer $(<20 \%)$ received the lowest number of answers with only $8.3 \%$ of the answers.

The questions regarding the guideline application score are described in this part of the analysis. Only four questions of 12 questions got $70 \%$ or more correct answers. The question that showed the lowest correct answers was the one concerned with the frequency of using CVD prevention on the patients (29.2\%).

The physicians were asked about the items they include in the written management plan. The answers included six items and each one of the items scored 1 mark in the total score; that is, including the full six items in the written plan, which means that the maximum number of marks is 6 . Only two items passed the $70 \%$ mark, namely, conditions where the patient is needed to go to the emergency room $(83.4 \%)$ and instruction for patients to avoid triggers (73.7\%).

The last question concerned the average rate of patients who have written CVD prevention management plans. This was a scaled question with the lowest percentage category associated with the lowest mark and the highest percentage category associated with the highest mark. An additional category was included in this question "I do not know." The answer in this category had no mark. Most of the physicians either did not know the answer $(44.2 \%)$ or did not answer $(<20 \%)$ which was the least correct answer $(42.7 \%)$.

In Table 3, the physicians' scores are stratified into two main categories (acceptable score, i.e., 70\% or above, and unacceptable score, i.e., <70\%). The results in the table show that physicians who scored acceptable marks in guidelines knowledge are only $14.2 \%$ (32/226). The application shows higher results in the satisfactory score with $48.7 \%(110 / 226)$ of the physicians having an acceptable score.

Table 3: Score stratification using the $70 \%$ mark as a cutoff point for acceptable scores versus unacceptable score ( $\mathrm{n}=\mathbf{2 2 6}$ )

\begin{tabular}{lll}
\hline Score & Score stratification & Frequency (\%) \\
\hline Knowledge & Acceptable & $32(14.2)$ \\
& Unacceptable & $194(85.8)$ \\
Application & Total & $226(100.0)$ \\
& Acceptable & $110(48.7)$ \\
& Unacceptable & $116(51.3)$ \\
& Total & $226(100.0)$ \\
\hline
\end{tabular}

The overall average scores of the physicians in knowledge and application score were compared with the minimum acceptable level. Table 4 shows the results of the comparisons using one-sample t-test. The results indicate that both average scores are statistically below the minimum acceptable level. The overall average score of knowledge is $56.5 \%$ which is well below the $70 \%$ mark. The application average score is $69.7 \%$ which is statistically not different from the $70 \%$ mark. 
Table 4: One-tailed, one sample t-test for testing knowledge and application scores versus the minimum acceptable score of $70 \%(n=226)$

\begin{tabular}{llllll}
\hline Score & Mean \pm SD & $\begin{array}{l}\text { Minimum } \\
\text { acceptance score }\end{array}$ & $\mathrm{P}^{* *}$ & \multicolumn{2}{l}{$95 \% \mathrm{Cl}$ of the difference } \\
\cline { 3 - 6 } & & & \multicolumn{2}{l}{ Lower bound } & Upper bound \\
\hline Knowledge & $56.5 \pm 13.6$ & 70 & $<.001$ & 54.9 & 58.1 \\
Application & $69.7 \pm 15.3$ & 70 & 0.071 & 67.8 & 71.5 \\
\hline
\end{tabular}

SD: Standard deviation; $\mathrm{Cl}$ : Confidence interval, ${ }^{* *}$ one-tailed, one-sample t-test with statistical significance level being 0.05

The determinants for knowledge among physicians were assessed using multiple linear regression. Physicians in the middle-aged group showed the highest knowledge when compared with their younger peers. Senior doctors also showed higher levels of knowledge when compared to younger FPs. The knowledge level without any of the factors was included in the model averaged $51 \%$ score. This model explained $28 \%$ of the variations among the physicians with regard to the differences in the physicians' knowledge of CVD prevention guidelines. Such a level of explanation can be considered moderate in the scope of health education assessment.

The level of application similarly was modeled using multiple linear regression. Female physicians had a statistically higher level of application score than male physicians. Senior doctors and consultants showed a higher level of application than FPs. Physicians who apply National guidelines had higher application scores than physicians who apply the European guidelines. Physicians who answered that they use guidelines other than National and European guidelines showed a statistically lower score. The model explained 35\% of the variation between physicians with regard to the application of guidelines. Again, this is considered a moderate explanation.

\section{Discussion}

This study found that knowledge and application of CVD prevention guidelines among FPs are low to average at best. The knowledge of the locally adapted guidelines is significantly lower than the European guidelines. There were differences among the physicians in knowledge and application of the guidelines, especially between junior doctors and their more senior colleagues. The physicians' gender, qualification, and position were associated with knowledge and application.

Based on our findings, we propose adapting or developing risk assessment tools that include patient factors, motivating behavioral changes in patients, and ensuring the adoption of cost-effective strategies for prescribing drugs. The wider use of absolute risk assessment tools and guidelines that directly take into account patient factors such as socioeconomic status, family history, and lifestyle choices may be more beneficial for FPs [9], [10], [11].
Motivating adherence for both behavioral and pharmaceutical changes remains a challenge for FPs. Despite that behavioral change is a highly effective prevention strategy, patient motivation and adherence to lifestyle recommendations are an obstacle to preventive care [12], [13]. A multifaceted approach at primary care settings, including controlled physical exercise, follow-up calls, and prescription reminders in addition to ongoing FP services, can improve patient adherence to prescribed medications and behavioral changes, while addressing barriers such as time and resource constraints for FPs [14], [15].

Our study also identified several research gaps, including prospects for assessing overall or individual risk, the effects of long-term drug dependence, primary care prescription recommendations, and gender and family support issues. The distinction between assessing absolute risk and individual risk factors is important to ensuring a more consistent and evidencebased evaluation of treatment plans. Current studies also did not examine primary prevention in detail and the necessity for the medication in asymptomatic patients [16], [17].

Raising awareness and adhering to evidencebased medication guidelines for asymptomatic patients and risk factors can improve consistency in assessing and managing the risk of CVD in patients. There was also a lack of information on the GPs thoughts on the role of family support. Family members can promote and support behavior change by encouraging prophylactic lifestyle choices and reminding patients to take medication [18], [19].

FPs believed that it was necessary to enable patients to prevent CVDs by following a lifestyle and taking medication, but it was difficult for them to stimulate behavior change. Some believed that clinical decision-making for the prevention of CVD was associated with the life stage and circumstances of the patient, self-control, and their environment, which were not taken into account in risk assessment and decisionmaking tools. The greater availability and adaptability of evidence-based strategies for assessing and managing the CVD risk, including changing patient behavior, can help in making decisions and taking measures to implement CVD prevention activities among FPs.

The results of our study should be interpreted in light of several limitations. First, the cross-sectional design limits the ability to make a causal inference. However, most of the predictors included in this study are factors that have not changed over time, and thus, the possibility of inverse causality is extremely low. Second, our survey was conducted among FPs only in Kharkiv Region. Our results are not representative of all FPs in Ukraine. In addition, we only measured one aspect of knowledge (CVD prevention knowledge) in this study. More dimensions of health literacy are needed to be included in future studies. In the end, the conclusion was based on self-reported data. Although the data 
were collected by experienced trained interviewers and the quality control procedure was used throughout the data collection and entry, we have no ways to control the potential information bias from FPs.

\section{Conclusions}

This study has identified genuine issues in CVD prevention management; only $14.2 \%$ showed good knowledge and application. The lack of knowledge and application of guidelines among physicians at primary care settings should be addressed more seriously; such an issue as the evidence from several studies suggests can be crucial in CVD prevention. This was apparent in young doctors with low experience.

It is necessary to expand the awareness of the availability of local guidelines and conduct future research to make sure that the guidelines are applied in different areas and FPs are aware of local guidelines .

\section{Ethics Approval}

The research protocol was presented to and approved by the Ethics Commission of the Kharkiv Medical Academy of Postgraduate Education of the Ministry of Health of Ukraine (Kharkiv, UA) (10/2018). The participants were informed that their participation is voluntary and they are free to refuse the participation in the study or withdraw at any stage without being asked about the reasons or being persuaded. The participants were informed that their refusal or withdrawal from the study participation has no consequences and their information will be confidential. The participants gave oral consent to participate in this study. .

\section{Acknowledgments}

The authors thank all the physicians who involved in the study and supported us in the research process.

\section{Data Availability Statement} the article.

All data relevant to the study are included in

\section{References}

1. Roth GA, Huffman MD, Moran AE, Feigin V, Mensah GA Naghavi M, et al. Global and regional patterns in cardiovascular mortality from 1990 to 2013. Circulation. 2015;132(17):1667-78. https://doi.org/10.1161/circulationaha.114.008720 PMid:26503749

2. Sasieni PD, Wald NJ. Should a reduction in all-cause mortality be the goal when assessing preventive medical therapies? Circulation. 2017;135(21):1985-7. https://doi.org/10.1161/ circulationaha.116.023359 PMid:28533316

3. Jansen J, McKinn S, Bonner C, Irwig L, Doust J, Glasziou P, et al General practitioners' decision making about primary prevention of cardiovascular disease in older adults: A qualitative study. PLoS One. 2017;12(1):e0170228. https://doi.org/10.1371/ journal.pone. 0170228

PMid:28085944

4. Piepoli MF, Hoes AW, Agewall S, Albus C, Brotons C, Catapano Al, et al. 2016 European guidelines on cardiovascular disease prevention in clinical practice: The sixth joint task force of the European society of cardiology and other societies on cardiovascular disease prevention in clinical practice (constituted by representatives of 10 societies and by invited experts): Developed with the special contribution of the European association for cardiovascular prevention and rehabilitation (EACPR). Eur Heart J. 2016;37(29):2315-81. https://doi.org/10.1093/eurheartj/ehw106

PMid:27222591

5. Heijmans N, van Lieshout J, Wensing M. Information exchange networks of health care providers and evidence-based cardiovascular risk management: An observational study. Implement Sci. 2017;12(1):7.

PMid:28086813

6. Byrne D, O'Connor L, Jennings S, Bennett K, Murphy AW. A survey of GPs awareness and use of risk assessment tools and cardiovascular disease prevention guidelines. Ir Med J. 2015;108(7):204-7

PMid:26349349

7. Unified Clinical Protocols of Primary, Secondary (Specialized) and Tertiary (Highly Specialized) Care (UKPMD). Prevention of Cardiovascular Disease; 2016.

8. Van Ness PH, Towle VR, Juthani-Mehta M. Testing measurement reliability in older populations: Methods for informed discrimination in instrument selection and application. J Aging Health. 2008;20(2):183-97.

PMid:18089767

9. Dyakova M, Shantikumar S, Colquitt JL, Drew CM, Sime M, Maclver $\mathrm{J}$, et al. Systematic versus opportunistic risk assessment for the primary prevention of cardiovascular disease. Cochrane Database Syst Rev. 2016;1:CD010411. https://doi.org/10.1002/14651858.cd010411.pub2

PMid:26824223

10. Chauhan U. Cardiovascular disease prevention in primary care Br Med Bull. 2007;81-82:65-79. PMid:17339273

11. Goff DC, Lloyd-Jones D, Bennett G, Coady S, D'Agostino RB Gibbons R, et al. ACC/AHA guideline on the assessment of cardiovascular risk: A report of the American college of cardiology/American heart association task force on practice guidelines. Circulation. 2013;129(25 Suppl 2):S49-73. PMid:24222018

12. Korzh $\mathrm{O}$, Krasnokutskiy S. Significance of education and self-management support for patients with chronic heart 
failure in family physician practice. Fam Med Prim Care Rev. 2016;18(4):432-6. https://doi.org/10.5114/fmpcr.2016.63697

13. Barfoed BL, Paulsen MS, Christensen PM, Halvorsen PA, Jarbøl DE, Larsen ML, et al. Associations between patients' adherence and GPs' attitudes towards risk, statin therapy and management of non-adherence a survey and register-based study. Fam Pract. 2016;33(2):140-7. https://doi.org/10.1093/ fampra/cmw005

PMid:26936208

14. Murphy E, Vellinga A, Byrne M, Cupples ME, Murphy AW, Buckley $\mathrm{B}$, et al. Primary care organisational interventions for secondary prevention of ischaemic heart disease: A systematic review and meta-analysis. Br J Gen Pract 2015;65(636):e460-8. https://doi.org/10.3399/bjgp15x685681

PMid:26120136

15. Korzh O, Krasnokutskiy S, Pankova O. Improving the drug compliance of hypertensive patients in primary care: Importance of health education and self-management. Arch Balk Med Union. 2019;54(3):497-502. https://doi.org/10.31688/abmu.2019.54.3.15

16. Khan N, Marvel FA, Wang J, Martin SS. Digital health technologies to promote lifestyle change and adherence. Curr Treat Options Cardiovasc Med 2017;19(8):60. https://doi. org/10.1007/s11936-017-0560-4

\section{PMid:28647844}

17. Cole JA, Smith SM, Hart N, Cupples ME. Do practitioners and friends support patients with coronary heart disease in lifestyle change? A qualitative study. BMC Fam Pract. 2013;14:126. https://doi.org/10.1186/1471-2296-14-126

PMid:23984815

18. Liew SM, Blacklock C, Hislop J, et al. Cardiovascular risk scores: Qualitative study of how primary care practitioners understand and use them. Br J Gen Pract. 2013;63(611):e401-7. https://doi. org/10.3399/bjgp13x668195 PMid:23735411

19. Tanner RM, Safford MM, Monda KL, Taylor B, O'Beirne R, Morris $\mathrm{M}$, et al. Primary care physician perspectives on barriers to statin treatment. Cardiovasc Drugs Ther. 2017;31(3):303-9. https://doi.org/10.1007/s10557-017-6738-x

PMid:28710589 